\title{
The choice of heart valve prosthesis for aortic valve replacement in the young: about choices and consequences
}

\author{
Thierry Bove \\ Department of Cardiac Surgery, University Hospital Gent, Gent, Belgium \\ Correspondence to: Thierry Bove, MD, PhD. Department of Cardiac Surgery, University Hospital of Gent, De Pintelaan 185, 5K12, 9000 Gent, \\ Belgium. Email: Thierry.bove@ugent.be. \\ Provenance: This is a Guest Editorial commissioned by Section Editor Busheng Zhang, PhD, MD (Department of Cardiac Surgery, Shanghai Chest \\ Hospital, Shanghai Jiaotong University, Shanghai, China). \\ Comment on: Schnittman SR, Adams DH, Itagaki S, et al. Bioprosthetic aortic valve replacement: Revisiting prosthesis choice in patients younger than \\ 50 years old. J Thorac Cardiovasc Surg 2018;155:539-47.e9.
}

Submitted Jan 22, 2018. Accepted for publication Feb 08, 2018.

doi: $10.21037 /$ atm.2018.02.22

View this article at: http://dx.doi.org/10.21037/atm.2018.02.22

In absence of the 'ideal' heart valve prosthesis, the debate on the optimal choice of valve substitute in patients requiring aortic valve replacement is ongoing. The controversy is even more alive when it concerns the younger population between 20 and 50 years of age.

Commonly, two types of valve prostheses are proposed. Mechanical valves are designed to last a lifetime but necessitate the lifelong intake of oral anticoagulants, thereby balancing between the risk of bleeding on one hand, and valve-related thrombo-embolism on the other hand, due to the narrow therapeutic regime. Biological valves are less interfering with daily life customs, but remain prone to structural deterioration over time, inevitably resulting in the need for reoperation in younger patients. In this paper, Schnittman et al. have been looking at mortality and morbidity outcome in young adults undergoing bioprosthetic versus mechanical aortic valve replacement (1). The study cohort was retrieved from the Health State Database of California and New York state, including all patients ageing between 18 and 50 years, who required a valve replacement for acquired non-infectious aortic valve disease, between 1997 and 2006. Originally 10,055 patients were identified, and after application of exclusion criteria and propensity score matching of the cohorts in function of the used prosthesis, two groups of 1,175 patients were retained for comparison. Considering that $2 / 3$ of the patient cohort was between 40 and 50 years of age, the survival over 15 years was identical for both prostheses, yielding neither any effect of the interaction between age and prosthesis type. However, the hazard risk of stroke and bleeding in the 15 -year follow-up, was lower in patients with a biological valve, being respectively $5.4 \%$ and $4.2 \%$ compared to $8.1 \%$ and $8.4 \%$ in patients with a mechanical prosthesis. In contrast, the cumulative risk of reoperation was increased in patients treated with a bioprosthesis, rising progressively from 2.2 at 5 years to 3.6 at 10 years and 5.9 at 15 years. Moreover, this risk was even more prominent when the study population was stratified by age group, showing a cumulative incidence of reoperation of $28 \%$ for patients between $18-30$ years, and $24.5 \%$ for patients between 41-50 years. From these data, the authors concluded that a bioprosthesis can be presented as a reasonable alternative to mechanical valve replacement, affording the perspective on a similar survival despite the risk of reoperation. The recommendation from this study is valid as a biological prosthesis is associated with a better safety profile and sustained lifestyle quality, by avoiding anticoagulation. Otherwise, the survival appears to be unaffected by the risk of structural degeneration and the need for reoperation, based on a commonly low 30-day mortality associated with a first reoperation. Contrarily, the main concern on the use of a mechanical prosthesis is the poor compliance to anticoagulation therapy in younger patients, entailing a constant risk of potentially life-threatening morbidities like stroke and major hemorrhage.

However, a few issues with possible impact on the valve- 
related outcome remain unaddressed in this paper, and require additional discussion at the time of decision-making on the appropriate aortic valve prosthesis.

(I) The primary benefit of mechanical valves is undoubtedly the maintenance of structural integrity, reducing thereby the need for late reoperation. The main drawback is related to the lifelong condemnation to take anti-coagulating drug agents, independently of the type of mechanical prosthesis. Our group demonstrated that an anticoagulation policy, targeting a lower INR of 1.5 to 2.5 , is safe and effective over more than 20 years, in selected patients with a mechanical aortic prosthesis, respecting that sinus rhythm and global left ventricular function are preserved (2). Such regimen allowed to reduce significantly the linearized risk of bleeding from $1.21 \%$ to $0.61 \%$ per patient/year, without increasing the risk of valve thrombosis or thrombo-embolic complication. Nonetheless, regular INR control is still mandatory as increased INR variability was an independent determinant of decreased survival and increased incidence of valve-related events in this population treated with a mechanical prosthesis. Therefore, empowering the patient's awareness of the consequences of this treatment like through advancing selfmonitoring of INR, should be stimulated when a mechanical prosthesis is proposed for aortic valve replacement (3).

(II) If a biological substitute is preferred, one has to consider that not all bioprosthetic valves are alike. Regarding durability, the CE-Perimount pericardial valve has shown to be the most reliable bioprosthesis in patients younger than 60 years (4). In a competing risk model, the expected valve durability before being subjected to a reoperation for structural degeneration (SVD), was in average 17.6 years in a commonly male population with a mean age of 51 years. But at the age of 35 years, the probability to have a valve reoperation was yet $70 \%$ at 15 years after implantation, confirming the equivocal relationship between patient age and bioprosthetic longevity. Hence, the results with other types of bioprosthesis such as the Mitroflow pericardial valve and the porcine Hancock II valve are certainly less convincing $(5,6)$. In addition, a smaller prosthesis size and consequently induced patient-prosthesis mismatch are important issues with a negative effect on the freedom from SVD (7). Both factors are known to accelerate stenotic and calcific degeneration of a bioprosthesis, and should be recognized at the time of aortic valve replacement in the young-especially in female patients with a small aortic root-through adapting an appropriate surgical technique or a different choice of prosthesis.

It is true that re-replacement of a dysfunctional aortic bioprosthesis is nowadays achieved with the same operative risk as the first-time surgery, but a substantial number of them will also require a second valve reoperation, often at a stage that these patients are older and present other comorbidities, compromising the reoperative outcome (8). Suggesting a transcatheter valve-invalve replacement is a valid alternative to surgical reoperation in the failed bioprosthesis. However, considering the little data on transcatheter valve durability, its real indication remains questionable, especially in the younger aged cohort (9-11).

(III) In the reported series, the age range varied from 18 to 50 years, carrying thereby a wide spectrum of life habits and expectations, as well as of interfering diseases across the age groups. Concurrent to the use of biological valve prostheses, the pulmonary autograft is an excellent alternative in these younger patients, affording the same advantages on quality of life. Moreover, many studies are reporting a long-term freedom from autograft-related reoperation of more than $90 \%$ at 15 years, clearly superior to the average biological valve durability in a comparable age group. In contrast, some intrinsic anatomical features like aortic annulus dilatation, and also the technical requirements at the time of implantation-both potentially jeopardizing the outcome of autograft valve function-are refraining the wider applicability of this procedure $(12,13)$.

Since the survival difference between prosthetic types for aortic valve replacement may be very subtle, the proper selection of the prosthesis is increasingly depending on factors as patient age and life expectancy, life style, pregnancy wish, and the willing to take medication. All these quality-of-life components need to be integrated into an engagement of shared decision-making concerning the final valve choice. Only well-informed patients, understanding the risks and benefits of the different options, are able to weigh these risks and benefits in contribution of 
their choice. Despite the mutual agreement before surgery, more than one third of the patients still feel post-hoc too poorly involved in the decision-making process, leaving them with uncertainties with negative impact on their well-being (14). According to a recent Dutch study, the application of a nation-wide online decision-aid protocol to support the patient's prosthesis type selection, is helpful to make the patient more knowledgeable and better informed regarding the valve options, thereby decreasing the anxiety and improving the mental health in front of the planned aortic valve surgery (15). Only through implementing such decision-aid system, one can improve the responsibility of the patient and alleviate the chance to get an eventual decisional conflict on the valve choice, once a particular choice-related consequence is interfering with the individual's well-being.

\section{Acknowledgements}

None.

\section{Footnote}

Conflicts of Interest: The author has no conflicts of interest to declare.

\section{References}

1. Schnittman SR, Adams DH, Itagaki S, et al. Bioprosthetic aortic valve replacement: Revisiting prosthesis choice in patients younger than 50 years old. J Thorac Cardiovasc Surg 2018;155:539-47.e9.

2. Bové T, Van Belleghem Y, François K, et al. Low targetINR anticoagulation is safe in selected aortic valve patients with the Medtronic Open Pivot mechanical prosthesis: long-term results of a propensity-matched comparison with standard anticoagulation. Interact Cardiovasc Thorac Surg 2017;24:862-8.

3. Dauphin C, Legault B, Jaffeux P, et al. Comparison of INR stability between self-monitoring and standard laboratory method: preliminary results of a prospective study in 67 mechanical heart valve patients. Arch Cardiovasc Dis 2008;101:753-61.

4. Bourguignon T, Bouquiaux-Stablo AL, Candolfi P, et al. Very long-term outcomes of the Carpentier-Edwards Perimount valve in aortic position. Ann Thorac Surg 2015;99:831-7.

5. Saleeb SF, Newburger JW, Geva T, et al. Accelerated degeneration of a bovine pericardial bioprosthetic aortic valve in children and young adults. Circulation 2014;130:51-60.

6. Une D, Ruel M, David TE. Twenty-year durability of the aortic Hancock II bioprosthesis in young patients: is it durable enough? Eur J Cardiothorac Surg 2014;46:825-30.

7. Flameng W, Herregods MC, Vercalsteren M, et al. Prosthesis-patient mismatch predicts structural valve degeneration in bioprosthetic heart valves. Circulation 2010;121:2123-9.

8. Chan V, Lam BK, Rubens FD, et al. Long-term evaluation of biological versus mechanical prosthesis use at reoperative aortic valve replacement. J Thorac Cardiovasc Surg 2012;144:146-51.

9. Grubitzsch H, Galloni M, Falk V. Wrinkles, folds and calcifications: Reduced durability after transcatheter aortic valve-in-valve replacement. J Thorac Cardiovasc Surg 2017;153:266-8.

10. Martin C, Sun W. Transcatheter valve underexpansion limits leaflet durability: implications for valve-in-valve procedures. Ann Biomed Eng 2017;45:394-404.

11. Van Steenberghe M, de Vasconcelos CY, Delay D, et al. Early transcatheter aortic valve degeneration in the young. Int J Cardiol 2016;222:786-7.

12. Charitos EI, Takkenberg JJ, Hanke T, et al. Reoperations on the pulmonary autograft and pulmonary homograft after the Ross procedure: An update on the German Dutch Ross Registry. J Thorac Cardiovasc Surg 2012;144:813-21; discussion 821-3.

13. Kalfa D, Mohammadi S, Kalavrouziotis D, et al. Longterm outcomes of the Ross procedure in adults with severe aortic stenosis: single-centre experience with 20 years of follow-up. Eur J Cardiothorac Surg 2015;47:159-67; discussion 67.

14. Korteland NM, Top D, Borsboom GJ, et al. Quality of life and prosthetic aortic valve selection in non-elderly adult patients. Interact Cardiovasc Thorac Surg 2016;22:723-8.

15. Korteland NM, Ahmed Y, Koolbergen DR, et al. Does the Use of a Decision Aid Improve Decision Making in Prosthetic Heart Valve Selection? A Multicenter Randomized Trial. Circ Cardiovasc Qual Outcomes 2017;10.

Cite this article as: Bove T. The choice of heart valve prosthesis for aortic valve replacement in the young: about choices and consequences. Ann Transl Med 2018;6(10):184. doi: 10.21037/atm.2018.02.22 